\title{
Analyse morphologique des mots composés de l'anglais juridique
}

\section{Barbara S. Villez}

\section{CpenEdition}

\section{Journals}

Édition électronique

URL : http://journals.openedition.org/asp/3735

DOI : 10.4000/asp.3735

ISSN : 2108-6354

\section{Éditeur}

Groupe d'étude et de recherche en anglais de spécialité

\section{Édition imprimée}

Date de publication : 1 décembre 1995

Pagination : 73-80

ISSN : 1246-8185

\section{Référence électronique}

Barbara S. Villez, «Analyse morphologique des mots composés de l'anglais juridique », ASp [En ligne], 7-10 | 1995, mis en ligne le 30 juillet 2013, consulté le 06 mai 2019. URL : http:// journals.openedition.org/asp/3735; DOI : 10.4000/asp.3735

Ce document a été généré automatiquement le 6 mai 2019.

Tous droits réservés 


\title{
Analyse morphologique des mots composés de l'anglais juridique
}

\author{
Barbara S. Villez
}

On reproche souvent à l'anglais juridique d'être un jargon obscur et archaïque, voire incompréhensible et maladroit. Les documents rédigés dans ce domaine sont caractérisés par un style très formel, chargé de mots et d'expressions spécialisés. En fait, l'anglais du droit est constitué d'une multitude de mots formés par dérivation morphologique, et en particulier de mots composés.

2 Les analyses morphologiques faites à ce jour ne s'occupent généralement que des mots usuels de l'anglais. Pour cette raison, il nous semble utile d'examiner les types de mots dérivés trouvés dans ce langage spécialisé et de vérifier si les règles de construction qui les gouvernent obéissent aux règles générales de la morphologie anglaise.

3 La morphologie, nous le rappelons, est l'étude de la formation des mots à partir des unités de sens indivisibles en unités plus petites. Les morphèmes, comme ils sont appelés, sont d'une part constitués de phonèmes qui font l'objet de l'analyse phonétique et d'autre part constituent des phrases, dont la structure fait l'objet de l'analyse syntactique. Tous ces éléments, bien que limités en nombre, permettent des combinaisons infinies. Ainsi, la personne initiée au système de la manipulation des morphèmes est capable de produire des mots et des phrases sans devoir les mémoriser au préalable. La production ou la création de la langue qui en découle est guidée par le sens que celui qui parle veut transmettre.

4 En anglais, le verbe work est un morphème car il porte un sens, mais il n'est pas divisible en d'autres éléments plus petits que lui et sémantiquement indépendants. Lorsqu'on ajoute à ce mot un autre morphème -ed signifiant le passé, on obtient un nouveau mot : worked, construit grâce à cette combinaison de morphèmes: work, la base (libre, car indépendante) et -ed, un suffixe (un morphème lié car inutilisable non attaché à un autre). Le résultat est une forme fléchie du verbe, work: il a une fonction syntactique différente de la base lorsqu'elle est employée seule, mais partage la même signification que celle du morphème libre, work. En revanche, si l'on affixe le morphème -er à droite de la même base, on obtient le mot: worker. La signification du résultat se trouve modifiée et sa 
catégorie grammaticale est différente de celle du morphème libre du départ ; d'un verbe, nous sommes passés à un substantif. Pour ces deux raisons, il s'agit cette fois d'un mot dérivé et non d'une flexion.

5 Les mots dérivés engendrés par l'affixation de morphèmes se révèlent très intéressants pour le locuteur en général parce qu'il y trouve un champ potentiel très riche de formation de mots. Plus intéressant encore, la dérivation morphologique assure souvent une économie d'éléments. En effet, a worker demande moins d'effort que a person who works et s'insère plus facilement dans tout type de phrase. La publicité sait tirer profit de cette économie qui véhicule souvent un impact plus efficace du message publicitaire. La marque Nabisco par exemple a choisi de nommer son nouveau gâteau sec, un sandwich de pâte d'arachide (peanut butter) : Nutter Butter. En conservant le radical nut du mot peanut, Nabisco préserve l'identification du type de produit exprimé par le composé peanut butter. L'emploi du morphème -er, qui signifie "plus ", communique un double "plus » aux consommateurs potentiels: qu'il y a plus d'arachides dans le nouveau biscuit et qu'ils sont better, (better butter jouant sur l'idée de redoublement [reduplication] morphologique [la répétition par les affixes de toute ou d'une partie de la base] que nous a laissée Sapir).

\section{Dérivation morphologique}

6 L'emploi que fait l'anglais juridique de la dérivation morphologique est également motivé par l'économie de mots qui en résulte, en même temps qu'une meilleure transmission de sens. En effet, un mot composé tel que wedlock est nettement plus concis que the bonds of marriage, mais ne sacrifie rien à la précision. Ceci est un point de convergence avec le langage juridique français qui utilise également un grand nombre de mots dérivés et de composés (un héritier réservataire, le blanc-seing, etc.).

7 Beaucoup de mots utilisés en anglais juridique sont des dérivés formés par affixation à une base (en-force-ment, afore-thought), ce qui est un procédé fréquent dans la langue usuelle. Le vocabulaire spécialisé retrouvé dans le domaine juridique en anglais est fondé surtout sur des mots composés qui se définissent comme étant constitués de plusieurs bases ou sur morphèmes libres, où chacun est un radical. Ainsi l'exemple ci-dessus mentionné wedlock est le résultat de la combinaison du verbe to wed (épouser) et le substantif lock (une serrure), produisant un substantif signifiant les liens du mariage. Un autre composé juridique est formé des verbes to hear et to say pour donner le substantif hearsay (un témoignage fondé sur le ouï-dire, son cousin du français juridique). De la même manière, le morphème judge se rattache à l'adjectif/base liée made (liée car elle ne peut servir d'adjectif tout seul) pour donner l'adjectif dérivé composé judgemade. Les exemples foisonnent : landlord, counterpart, copyright, et manslaughter, et bien sûr toute l'énorme famille des repères dans l'espace et dans le temps qui sont des mots tels que: herein, aforementioned, thereinunder, wheretofore et ainsi de suite.

8 La dérivation produit donc un grand nombre de composés juridiques ayant les fonctions de substantifs, de verbes, d'adjectifs et d'adverbes. D'autres formes de composés existent qui ne sont pas attachés mais sont des collocations forgées qui fonctionnent comme un seul mot, tels que specific performance, implied terms, duty of care, breach of contract, land charges et summary trial. Suivant les règles morphologiques générales, la fonction sémantique de ces expressions peut être déduite de la signification des parties composantes, tout au moins pour des juristes de langue anglaise. Il diffère en cela des 
expressions qui, selon A. M. Di Sciullo et E. Williams (1987), nécessitent un recours au dictionnaire car ils sont idiomatiques. En effet lorsqu'on trouve implied terms dans un contexte contractuel, il est facile de comprendre "clauses impliquées", mais rien ne permettrait de déduire la signification juridique du verbe to leapfrog (passer directement à la cour supérieure) et comment arriver à la notion du reasonable man (le bon père de famille) à partir de the man on the Clapham omnibus?

Pour clore cette réflexion sur les types de dérivés trouvés en anglais juridique, il faut mentionner une petite catégorie de mots qui subissent l'affixation du morphème -s, habituellement la marque du pluriel en anglais, mais qui dans ces cas produit plutôt une divergence sémantique. Ainsi, damages ne signifie point le pluriel de damage (dommage), mais la compensation financière (dommages et intérêts) versés au demandeur qui intente l'action. Parallèlement, pleadings sont des documents rédigés par un barrister en préparation d'un procès et non le pluriel de l'activité de plaidoirie (pleading).

\section{Affixation}

10 La linguistique moderne établit une hiérarchie d'ordre gouvernant l'affixation. Si les morphèmes -less et -ness sont tous les deux présents dans un mot, le premier sera attaché à la base avant le second. Le premier bloque l'affixation du second qui ne pourra s'y attacher qu'après. Par conséquent, hopelessness est possible tandis que hopenessless est inacceptable. De la même manière, démontre F. Katamba (1993: 123) dans le cas des pluriels irréguliers, le morphème représentant le pluriel le moins général et par extension le moins productif, bloque le morphème -s qui est la marque du pluriel la plus générale et donc la plus productive. Par exemple, pour person, l'allomorphe people est appliqué en priorité par rapport au morphème - s, ce qui produit un autre cas de divergence sémantique. People sert de pluriel pour a person (une personne) dans la langue courante et persons est réservé au pluriel des individus, par exemple dans des rapports contractuels.

11 Malgré la présence de dérivés de différentes sortes en anglais juridique, le grand nombre de composés mérite que l'on s'attarde sur leur construction et les règles de lecture qui s'y appliquent. Il serait logique de supposer que ces mots obéissent aux règles générales de l'analyse morphologique des composés. Si le procédé de composition vise une productivité ouverte, des généralisations seraient utiles.

12 Tout mot composé possède au moins un morphème qui est considéré comme la tête. Selon certaines théories linguistiques, la tête peut être grammaticale, déterminant alors la fonction syntaxique du mot dérivé (-ness serait dans ce cas la tête grammaticale du mot hopelessness distribuant aux autres morphèmes la qualité de substantif). D'autres linguistes préfèrent considérer que la tête remplit un rôle sémantique et dans ce cas il est le morphème qualifié par les autres dans le mot composé. Dans les mots understatement, word order et crossroads, les têtes sont l'élément dominant, ce dont il s'agit, c'est-à-dire : statement, order, roads. Il semblerait qu'en anglais la tête se trouve la plupart du temps être l'élément à la droite du mot composé selon le Right-hand Head Rule de Williams (1981). De toute façon, le composé est endocentrique, quelle que soit la position de la tête, c'està-dire que la tête se trouve dans le mot ou dans l'expression. Dans certains cas, il y a des composés sans aucun élément dominant qui sont exocentriques, par exemple le composé hearsay. 
13 La position de la tête d'un mot composé est utile à la lecture, ou plutôt à l'analyse sémantique, par conséquent à la compréhension de ces mots souvent complexes, particulièrement dans le langage juridique. Les têtes des mots composés en italien et en français se trouvent généralement à gauche (Katamba 1983 : 315-316). « Un essuie-main », « un lit gigogne », « un héritier réservataire », « les frais de justice » ne contredisent pas cette affirmation. On comprend donc un objet pour s'essuyer les mains, une sorte de lit, un type d'héritier. L'interprétation va vers la tête, bien que les messages essentiels, à savoir le sens, la fonction syntactique soient répercutés à partir de la tête.

Lorsqu'on examine les composés de l'anglais juridique de près, on découvre que certaines classes grammaticales ont bien la tête à droite, ainsi convergeant avec les règles générales de la formation des mots composés. Cependant d'autres classes ont la tête à gauche et divergent totalement de ces règles (voir l'annexe). Ces mots, principalement les adverbes, les repères de temps et de lieu, se "lisent» donc de la droite vers la gauche. Par conséquent, hereinafter se traduit after (what is said) in here (le contrat), et herewith, with (by virtue of) (what is agreed) here, etc.

15 Il est frappant de remarquer qu'en français les adjectifs composés dans le langage juridique divergent de la règle de la tête à gauche pour cette langue. Les adverbes « tête à gauche » sont cohérents avec la règle et on constate la convergence avec les adverbes composés en anglais juridique. La confusion se dissipe lorsque l'on découvre que ces adverbes sont apparus à environ la même époque, c'est-à-dire le $\mathrm{X}^{\mathrm{e}}$ siècle pour " susvisé " du latin susum, sursum, le XII ${ }^{\mathrm{e}}$ pour «ci » («ci-dessus»), et le $\mathrm{X}^{\mathrm{e}}$ pour le début des composés à base de here. Here "en combinaison avec d'autres adverbes ou des prépositions comme dans d'autres langues germaniques (... est le résultat) de la juxtaposition de here et d'un autre adverbe qualifiant le même verbe. Herebefore est apparu au début comme haer beforan, «ici dans ce document", avant d'évoluer vers le sens de « un endroit plus tôt » (Oxford English Dictionary : 1933, 236).

R. Munday (1991) éclaircit le dilemme de savoir si les Anglais ont pris les adverbes de repère des Français ou si les Français ont rapporté des adjectifs composés d'outreManche. Il est vrai que l'anglais juridique peut être également caractérisé par la présence d'un nombre considérable de mots d'origine française et latine. En fait, selon Munday, le français, "déjà langue courante chez les nobles » anglais au XIII ${ }^{\mathrm{e}}$ siècle est devenu la langue utilisée devant les cours de justice en Angleterre. Cette situation ne dura qu'un siècle, mais les lois continuèrent à être rédigées en français, ainsi que les arrêts anglais jusqu'au XVII siècle. Le français resta donc la langue écrite de la cour de justice longtemps après qu'il eut cessé d'être parlé par les juristes anglais. Munday soutient que, malgré le fait que les Anglais le rendaient progressivement plus anglais, le français était pour eux difficile à abandonner parce qu'ils avaient leurs habitudes d'expression. L'utilisation du français leur apportait la précision réconfortante d'un langage spécialisé et une sorte de protection contre une trop grande limpidité pour les non-initiés.

17 Ce langage juridique ou "Law French" évolua en une langue méconnaissable pour les véritables Français. Certaines transformations doivent être la source des nombreux fauxamis existant entre le français juridique et l'anglais juridique (entre autres : jurisdiction/ juridiction, injunction, magistrate, jurisprudence). Munday cite plusieurs exemples de ce qu'est devenu le "Law French", dont le suivant (qui contient un composé) :

al common ley home poet user quel trade il voile, cybien un come auter: mes ne poet usar deux trades ensemble, ne un trade pur exerciser de quel il fuit insufficient. (1623) 
18

$$
\begin{aligned}
& \text { apportaient peu à peu des transformations à chaque fois qu'ils les adaptaient à un cas } \\
& \text { précis. Cette situation étant parallèle à celle que nous connaissons aujourd'hui, car ceux } \\
& \text { qui incriminent le langage lourd et complexe de l'anglais juridique militent pour } \\
& \text { l'utilisation d'un langage simple, direct et compréhensible par tous, y compris par les } \\
& \text { clients «The Plain English Movement for Lawyers » (Wydick 1985). L'utilisation maintenue } \\
& \text { des mots composés et notamment les adverbes étudiés ici est très contestée. Des } \\
& \text { entreprises, des assureurs, des clients ont fait pression sur leurs avocats pour qu'ils } \\
& \text { rédigent en Plain English. }
\end{aligned}
$$

Les Anglais devaient utiliser des documents rédigés en "Law French" comme modèles et y

\section{Conclusion}

Cependant les juristes se montrent linguistiquement conservateurs. Beaucoup d'entre eux éprouvent de la difficulté à écrire de manière exacte et rigoureuse sans les mots que la dérivation morphologique a fournis jusque là. Ils craignent de ne pouvoir rédiger des documents aussi bien protégés contre des détournements de sens (airtight legal documents ). Il est vrai aussi que, face à la concurrence actuelle qui se manifeste dans le monde juridique anglo-saxon, le fait de maintenir ce langage fondé sur des termes composés assure pour plus longtemps le besoin qu'éprouve le public de s'adresser à des juristes. Finalement, de même que les Anglais se servaient des documents en « Law French » au XVI ${ }^{e}$ siècle, les avocats internationaux d'aujourd'hui se servent de modèles de contrats et d'autres documents anglais déjà utilisés. Par ce fait, les mots composés typiquement juridiques ne sont pas près de disparaître. Peut-être évolueront-ils aussi vers des formes erronées un jour ; surtout s'ils ne sont jamais enseignés.

\section{BIBLIOGRAPHIE}

Di Sciullo, A.M. et E. Williams. 1987. On Defining the Word. Cambridge MA : MIT Press.

Katamba, F. 1993. Morphology. Londres : Macmillan.

Munday, R. 1991. « La langue française et la common law ». La Vie judiciaire 2366, 1-8.

Oxford English Dictionary (The) vol. 5. 1933. Oxford : Clarendon Press.

Williams, E. 1981. « On the notions 'lexically related' and 'head of a word' ». Linguistic Inquiry 12, 234-274.

Wydick, R.1985. Plain English for Lawyers. Durham, NC : Carolina Academic Press.

\section{Bibliographie complémentaire}

Baleyte, J. et al. 1984 [1977]. Dictionnaire juridique (Quemner) français-anglais. Paris : Navarre.

Black, H.C. 1983. Black's Law Dictionary. St. Paul, MINN : West Publishing Co.

Dubois, J. et al. 1989. Dictionnaire de linguistique. Paris : Larousse.

ASp, 7-10 | 1995 
Harrap's Dictionary of law and society. 1989. Kent : Harrap Books Ltd.

Matthews, P. 1974. Morphology. Cambridge : Cambridge University Press.

Robert, P et A. Rey. 1991. Dictionnaire de la langue française, vol. 2 et vol. 9. Paris : Le Robert.

\section{ANNEXES}

\section{Exemples de mots composés}

Les exemples de mots composés d'anglais juridique qui suivent sont classés selon leur structure (p. ex. nom + nom = nom, adjectif + nom $=$ nom, etc.). Toutes les têtes sont soulignées.

\section{Substantifs endocentriques :}

$n+n=n$

landlord, manslaughter, tortfeasor, copyright, common law marriage, land charge, lease

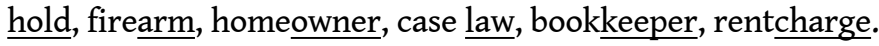

$\operatorname{adj}+n=n$ ou $n+\operatorname{adj} .=n$

counterclaim, goodwill, freehold, intellectual property, strict liability, blackmail, hung jury, smallholding, quiet enjoyment, case stated, fee simple.

prép $+n=n$

undertaking, underlease, aforethought, foreclosure

$v+n=n$

wedlock, search warrant

$a d v+v=n$ ou prép $+v=n$

welfare, offspring

$n+$ prép $+n=n^{*}$

abuse of process, act of god, contempt of court, power of attorney, grant of probate, duty of care, examination-in-chief, rule of law, breach of contract

\section{Substantifs exocentriques :}

$v+v=n$

hearsay, hire-purchase

Substantifs par la divergence sémantique :

damages/damage, pleadings/pleading, persons/people, acquittance/acquittal, accessions/accession

Verbes :

prép $+v=v$

to foreclose, to override, to undersign, to forswear

$a d v+v=v$ ou $a d j+v=v$

to backdate, to countersign, to cross-examine

adj $+n=v$ ou $n+v=v$ ou $v+n=v$

to blackball, to blacklist, to plea bargain, to leapfrog**

$v+$ prép $=v^{*}$

to hand down, to cover up 
Adverbes* :

$a d v+$ prép $=a d v$

herein, hereafter, hereinafter, hereunto, hereby, heretofore, whereby, wherefore, where in, theretofore, therefrom, thereof, therefor, ${ }^{* * *}$ henceforth, whencesoever

Adjectifs :

$a d v(a d v+$ prép $)+a d j=a d j$ ou pron $+a d v=a d j$

hereaftermentioned, hereinabovementioned, thereinundernamed, wheregot, wheregone, hereinbeforementioned, heretoforeagreed, whatsoever**

prép + adj. $=$ adj

aforsaid, after-acquired, aforementioned

adj + adj $=$ adj ou $n+a d j-a d j$

extraterritorial, feeble-minded, judgemade

* Il semblerait que la présence de prépositions dans la formation des composés ait une influence sur la position de la tête dans certains cas, par exemple la structure "latine" : nom + prép + nom (e. g. the burden of proof).

** Ces deux exceptions à "la règle de la tête à droite", tiennent certainement de la nature grammaticale de leurs constituants $(v+n=v ;$ pron $+a d v=a d j)$, mais ceci resterait à vérifier grâce à d'autres exemples de ce type.

*** therefor : à ne pas confondre avec therefore

La position de la tête révèle non seulement le sens de la lecture des mots composés, mais également l'ordre à respecter dans la formation de tels mots. Ainsi hereinabove est possible, indiqué par le sens de la lecture, mais hereabovein n'est pas acceptable. En effet la production des mots composés, et notamment des adverbes de l'anglais juridique est illimitée et par conséquent ces derniers ne figurent pas systématiquement dans les dictionnaires généraux ou spécialisés.

\section{RÉSUMÉS}

On reproche souvent à l'anglais juridique d'être un jargon obscur et archaïque, voire incompréhensible et maladroit. Les documents rédigés dans ce domaine sont caractérisés par un style très formel, chargé de mots et d'expressions spécialisés. En fait, l'anglais du droit est constitué d'une multitude de mots formés par dérivation morphologique, et en particulier de mots composés. Les analyses morphologiques faites à ce jour ne s'occupent généralement que des mots usuels de l'anglais. Pour cette raison, il nous semble utile d'examiner les types de mots dérivés trouvés dans ce langage spécialisé et de vérifier si les règles de construction qui les gouvernent obéissent aux règles générales de la morphologie anglaise.

\section{INDEX}

Mots-clés : anglais juridique, droit, morphologie, mot composé

Keywords : compound word, English for law, law, morphology 
AUTEUR

BARBARA S. VILLEZ

Barbara S. Villez enseigne à l'Université Panthéon-Assas Paris 2. barbara.villez@gmail.com 